\title{
Nanocluster Ions and Beam Techniques for Material Modification
}

\author{
Gikan H. Takaoka \\ Photonics and Electronics Science and Engineering Center, Kyoto University \\ Katsura, Nishikyo-ku, Kyoto 615-8510, Japan \\ Fax: 81-75-383-2343, and/or e-mail: gtakaoka@kuee.kyoto-u.ac.jp
}

\begin{abstract}
Various kinds of clusters such as hydrogen, ionic, and metallic bonding clusters were generated using several unique methods such as the nozzle beam, high-electric-field, and evaporation-on-liquid methods. The size and structure of these clusters were analyzed by time-of-flight (TOF), high-energy electron diffraction (HEED), transmission electron microscopy (TEM), and small-angle X-ray scattering (SAXS). In addition, the impact of the cluster ions on a solid surface was investigated, and unique irradiation effects were found, such as the low-energy irradiation effect and the high-density irradiation effect. The kinetic energy of the cluster ion was converted to thermal energy, resulting in extremely high temperatures. Furthermore, the simultaneous use of chemical sputtering and thermal annealing processes was demonstrated with ethanol cluster ion beams. As a result, low irradiation damage and high-rate sputtering of Si surfaces was performed by the retardation potential method.
\end{abstract}

Key words: Cluster, Ion beam, Vacuum, Irradiation effect, Material processing

\section{INTRODUCTION}

A nanocluster, which includes small and large clusters, is defined as a tiny particle on a mesoscopic scale with a diameter of approximately 1-10 nm. Such a nanocluster can form a link between the atomic state and the bulk state [1-4]. Furthermore, a large cluster is an aggregation of atoms (or molecules) that occurs as an isolated particle with a diameter of a few nanometers. These clusters reveal a new phase relative to the initial state of the materials, and the physical and chemical properties of a cluster are different from those of the bulk state [5-7]. Therefore, cluster studies have attracted much interest, and various kinds of cluster ions have been studied as an extension of new materials studies [8,9].

Ions and electrons are well known as charged particles, which offer the possibility of excellent controllability with regard to variables. For example, the kinetic energy of ions can be controlled by changing the acceleration voltage. Furthermore, it is possible to control, as well as to count, the number of ions by controlling the ion current.

In addition, an ion is a many-electron particle, which represents the abundant varieties of ion species [10-15] For example, many kinds of ions, as well as several charged states of ions, are available. In other words, positive or negative ions, as well as singly charged or multiply charged ions, are available, even of the same element. Furthermore, various ions such as atomic, molecular, polyatomic, and cluster ions are available according to the different electronic orbitals of the constituent atoms.

A cluster ion is a polyatomic particle, and the impact of the cluster ion on a solid surface result in a non-linear irradiation effect [16-19]. The kinetic energy of a cluster ion can be converted to thermal energy by cluster ion irradiation [20,21]. This effect is obtained by multiple collisions and the high-energy density irradiation of the cluster ion; it cannot be obtained by conventional monomer ion irradiation. Furthermore, these clusters can be generated using solid, liquid, or gas materials.

In general, a liquid material in a vacuum is regarded as "dirty," and it is considered a source of contaminants such as hydrocarbons and water molecules. Little attention has been paid to the usage of liquid materials in a vacuum. However, liquids at negligibly low vapor pressures, e.g. ionic liquids (ILs), have recently been used as a matrix (substrate) for metal deposition [22,23]. In addition, liquid materials such as water and alcohol have been used as solvents in organic and biological reactions. For example, ethanol has been applied in the surface treatment of solid surfaces in conventional wet processes. Chemical reactions such as substitution or elimination of the hydrophobic group in ethanol are different depending on the role of the hydroxyl substituent. Furthermore, in the case of liquid cluster ion beams, material modification and sputtering have been performed by adjusting the kinetic energy of the cluster ion [24-26]. In particular, radicals contained in a liquid molecule have attracted much interest in terms of chemical reactions on the surface. Thus, the liquid cluster ion beam technique plays an important role in advanced material processing as a new way of using liquid materials [27].

In this paper, the author reviews various unique methods of using liquid materials to generate nanoclusters. Clusters such as metallic, ionic, and hydrogen bonding clusters were generated, and the size and structural analysis of these clusters were performed. Furthermore, the cluster ion irradiation effects are discussed based on Rutherford backscattering spectrometry (RBS) and photo-luminescence measurements. In addition, chemical modification and sputtering processes were demonstrated with ethanol and water cluster ion beams, and their characteristics are investigated from the viewpoint of exotic ion beams.

\section{CLUSTER GENERATION}

\subsection{Experimental procedure}

Metallic, ionic, and hydrogen bonding clusters were produced using methods such as the nozzle beam, high-electric-field, and evaporation-on-liquid methods. A liquid cluster ion beam system was developed using the nozzle beam method, the details of which have been described elsewhere $[28,29]$. Liquid materials such as 
ethanol and water were placed in a metal box and heated by a wire heater attached around the source. The vapors of the liquids were ejected through the nozzle into a vacuum region. When the vapor pressure reached $1 \mathrm{~atm}$, vaporized clusters were produced by an adiabatic expansion phenomenon. In the system, the produced nanoclusters passed through a skimmer and a collimator, and then entered an ionizer. Inside the ionizer, the neutral clusters were ionized by electron bombardment. The cluster ions were accelerated by applying an extraction voltage to the extraction electrode. The extracted cluster ions were separated according to their size using the retardation potential method. The clusters used were larger than 100 molecules per cluster, which is a typical minimum size. Cluster ion beams with the large clusters were accelerated toward a substrate placed on a substrate holder. A background pressure of $6.0 \times 10^{-7}$ Torr was maintained around the substrate using a diffusion pump.

An ILs ion beam system was developed using the high-electric-field method, the details of which have been described elsewhere [30,31]. A high electric field was applied to a sharp tip of the needle to produce ion emission. The needle was made of carbon because it exhibits better wettability for ILs than $\mathrm{W}$ and stainless steel (SUS). In addition, carbon is an excellent material for use in mechanical processing. The diameter of the needle was $0.3 \mathrm{~mm}$. As a tip designed, for example, a needle with a radius of $80 \mu \mathrm{m}$ was employed. The needle was supported on a porous electrode covered with carbon felt and biased either positively or negatively with respect to a nearby aperture electrode that served as the extractor. The ionic liquid ions were emitted from the tip by applying the voltage between the tip and the extractor. As opposed to most ion sources with this method, either positive or negative ion beams can be extracted from IL sources depending only on the direction of the applied electric field, i.e., by selecting the appropriate polarity of the voltage source. The extracted ion beams were accelerated toward the substrate. The background pressure around the substrate was $1.0 \times 10^{-6}$ Torr, which was attained using a diffusion pump.

We developed a vacuum deposition system that uses various ILs as the matrix for metal deposition using the evaporation-on-liquid method, the details of which have been described elsewhere [32]. Briefly, a Au wire was hung on a W filament and heated by resistive heating. Au vapors were deposited onto the IL, which was contained in a glass petri dish. The deposition rate was monitored with a quartz crystal oscillator. The Au film thickness was kept constant at $100 \mathrm{~nm}$, and the Au density in the IL was approximately $0.04 \mathrm{wt} \%$. During deposition, some $\mathrm{Au}$ atoms were able to float on the IL surface owing to the relatively large surface tension, whereas other $\mathrm{Au}$ atoms and small nuclei sank below the IL surface. Nucleation growth occurred by the absorption of $\mathrm{Au}$ atoms into the nucleus that was formed. A background pressure of $1.0 \times$ $10^{-6}$ Torr was maintained around the glass dish using a turbo molecular pump.

\subsection{Cluster size and structure analysis \\ (a) Hydrogen bonding cluster}

The cluster size was measured by the time-of-flight (TOF) method [27]. As shown in Figure 1, the cluster size was distributed between a few hundred molecules and a few tens of thousands of molecules. The average size (peak size) was approximately 500 to 1,000 molecules for the ethanol and approximately 2,500 molecules for the water. The beam intensity of the cluster ions increased with increasing vapor pressure. According to thermodynamics and gas dynamics, the cluster beam intensity increases with increasing vapor pressure [33,34]. With regards to the minimum size of cluster ions in the size distribution, the retarding voltage was applied to remove monomer and small cluster beams. For example, the retarding voltage corresponding to a minimum size of 100 molecules was $14 \mathrm{~V}$ for water cluster ions and $28 \mathrm{~V}$ for ethanol cluster ions. Figure 2 shows the cluster size distribution for ethanol clusters at different retarding voltages. The minimum size increased with increasing retarding voltage $\left(V_{\mathrm{r}}\right)$. In addition, the peak size shifted to a higher value.
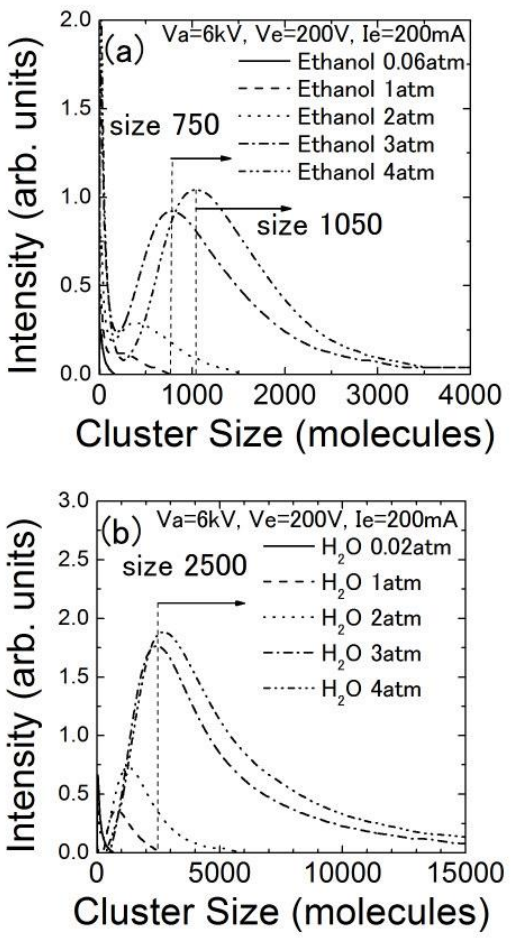

Fig. 1: Cluster size distribution for (a) ethanol clusters and (b) water clusters at different vapor pressures.

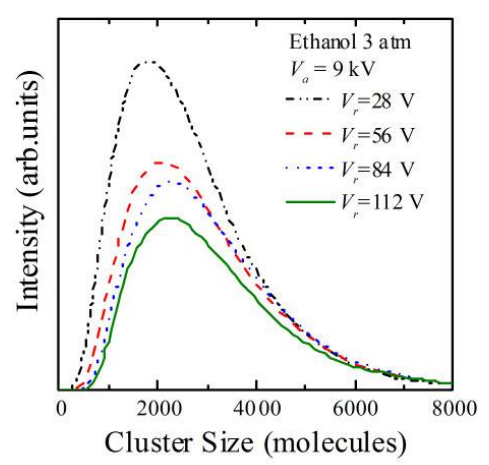

Fig. 2: Cluster size distribution for ethanol clusters at different retarding voltages. 
The high-energy electron diffraction (HEED) patterns of water cluster beams were obtained using an electron gun (ULVAC-PHI Corp., Type MB1000) installed in the cluster beam system. Figure 3 shows a typical diffraction image and diffraction patterns of water clusters generated at different vapor pressures. Debye-Scherrer rings are observed in Figure 3(a). The vapor pressure $\left(P_{0}\right)$ was 4.0 atm. Furthermore, several peaks corresponding to the (111), (220), (311) and (400) planes are observed and the peak position is almost the same at different vapor pressures. This indicates that the Debye-Scherrer rings correspond to an ice structure (Type $\mathrm{I}_{\mathrm{c}}$ ). Thus, the crystal state of the water cluster is different from the amorphous state of liquid water. In addition, the water cluster might consist of a networked structure of hydrogen bonds.

As an extension of water clusters with hydrogen-bridge networks, gas hydrate clusters have attracted much interest. Gas hydrates exhibit many features that are advantageous for a number of applications, such as flow assurance, safety, energy recovery, gas storage or transportation, and climate change [35]. Gas hydrates, also called clathrate hydrates, are crystalline compounds of water and gas molecules such as methane or carbon dioxide [36,37]. The inclusion of gas molecules into water cluster cages with a specific number of cluster sizes could be performed by introducing gas into liquid water for the cluster formation. In the previous study, water vapor mixed with methane or carbon dioxide gas was ejected through a nozzle into a vacuum region, and the gas-hydrate clusters were produced by adiabatic expansion $[38,39]$.

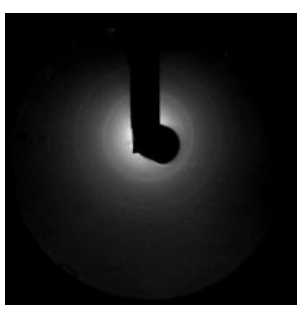

(a) $P_{0}=4.0$ atm

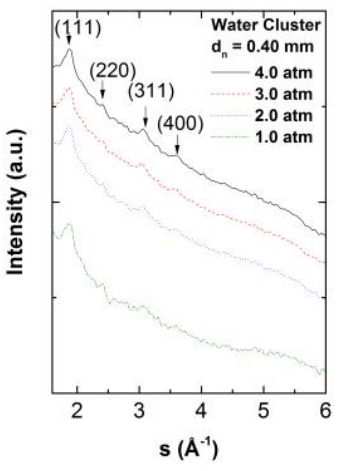

(b)
Fig. 3: (a) Typical diffraction image and (b) diffraction patterns of water clusters generated at different vapor pressures.

\section{(b) Ionic bonding cluster}

The cluster size was measured by the TOF method. To make TOF measurement, the accelerated cluster ions first entered a flight tube, and they were deflected by a negative voltage pulse. Due to the application of this voltage, the cluster ions drifted towards a Faraday cup mounted at the end of the flight tube, which detected the ions by a Micro Channel Plate (MCP). The acceleration voltage was changed from 3 to $4 \mathrm{kV}$, and the pulse voltage was adjusted between 1 and $2 \mathrm{kV}$. The duration time and the repetition rate of the pulse voltage were $5 \mu$ s and 1 $\mathrm{kHz}$, respectively. The drift distance was $1.7 \mathrm{~m}$.

Figure 4 shows the mass spectra for positive and negative IL ion beams. The IL used was 1-butyl-3-methylimidazolium hexafluorophosphate (BMIM-PF 6 ). The extraction voltage $\left(V_{\text {ext }}\right)$ was $4 \mathrm{kV}$. As shown in the figure, several peaks appeared in the mass spectra for positive and negative ions, although the mass resolution was not sufficient at higher masses. The molecular weight of the cation $\left(\mathrm{C}_{8} \mathrm{H}_{15} \mathrm{~N}_{2}\right)$ and the anion $\left(\mathrm{PF}_{6}\right)$ was 139.22 and 144.96, respectively. Both cations and anions were produced as positive and negative fragment ions. Furthermore, by assuming that singly charged ions were extracted, positive and negative cluster ions consisting of a few molecules to a few tens of molecules in size were produced. It has been reported that multiply charged clusters could be produced by using a salt with higher ionic bonding [40,41]. However, TOF measurements cannot distinguish a singly charged cluster with mass $m$ from a doubly charged cluster with mass $2 m$. Therefore, we assumed that singly charged ions were extracted.

In addition, IL clusters have ionic bonding ability, and the same number of cations and anions form a neutral IL cluster. For example, cation $\mathrm{A}^{+}$or anion $\mathrm{B}^{-}$can be attached to the neutral cluster $(\mathrm{AB})_{n}$, which results in the formation of a positive cluster ion $(\mathrm{AB})_{n} \mathrm{~A}^{+}$or a negative cluster ion $(\mathrm{AB})_{n} \mathrm{~B}^{-}$, both with a cluster size of $n$. The composition of IL layers deposited by positive and negative cluster ion beams was measured using X-ray photoelectron spectroscopy (XPS). The results were similar to that of an IL BMIM-PF 6 solvent [42]. This indicates that either a cation or an anion is attached to the neutral BMIM-PF 6 cluster resulting in the formation of positive or negative cluster ions, respectively.
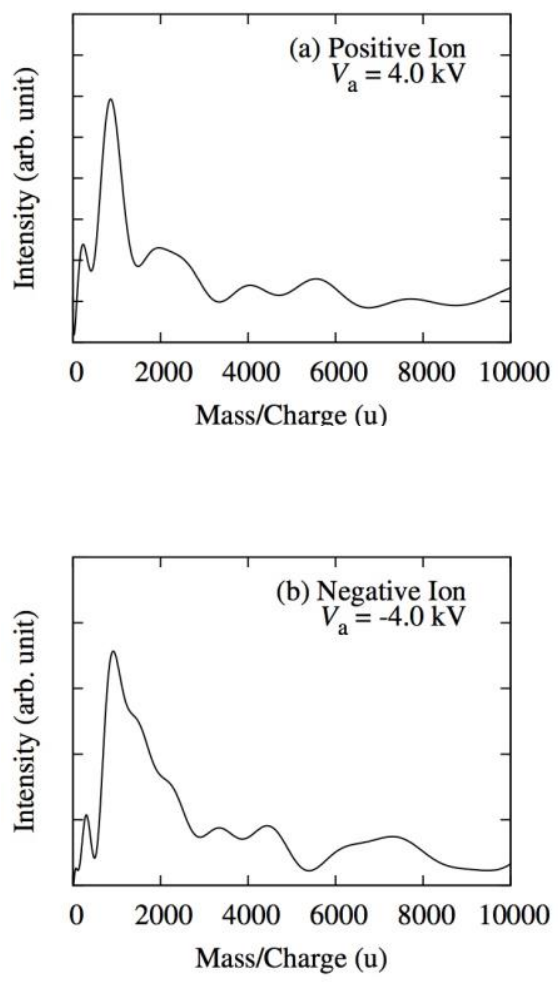

Fig. 4: Mass spectra for (a) positive and (b) negative IL ion beams. 
(c) Metallic bonding cluster

$\mathrm{Au}$ clusters were generated in ILs by the evaporation-on-liquid method. Transmission electron microscopy (TEM) observation of the Au clusters was performed, and fast Fourier transform (FFT) images were obtained. Figure 5 shows TEM and FFT images of Au clusters formed in the IL at deposition rates of 1.0 and 5.0 $\AA /$ s. The IL used was 1-ethyl-3-methyl-imidazolium dicyanamide (EMIM-DCA). The diameter of the $\mathrm{Au}$ clusters was approximately $5 \mathrm{~nm}$ when they were deposited at $1.0 \AA / \mathrm{s}$ and approximately $3 \mathrm{~nm}$ when they were deposited at $5.0 \AA / \mathrm{s}$. The large clusters had an fcc crystal structure, whereas the small clusters had a decahedral structure, which is different from the fcc structure of bulk Au.
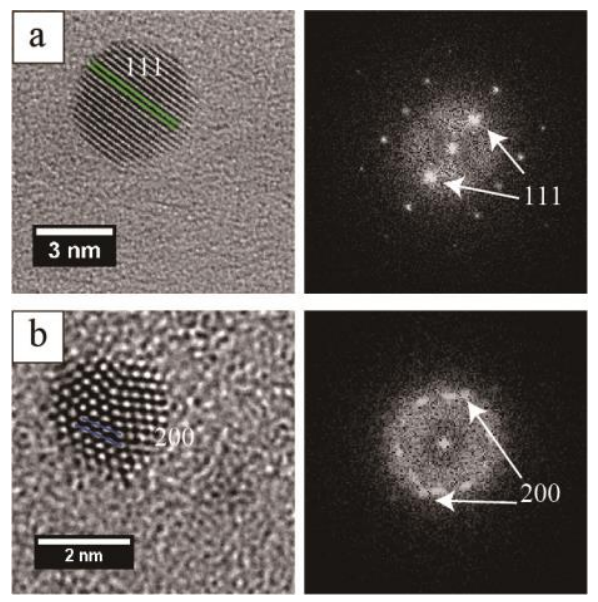

Fig. 5: TEM and FFT images of $\mathrm{Au}$ clusters formed in the IL at deposition rates of (a) 1.0 and (b) $5.0 \AA / \mathrm{s}$.

The size distribution of the $\mathrm{Au}$ clusters formed in EMIM-DCA was calculated by curve fitting of the small-angle X-ray scattering (SAXS) profiles. As shown in Figure 6 , the cluster size decreased with increasing deposition rate. The diameter of the $\mathrm{Au}$ clusters was distributed between approximately 1 and $6 \mathrm{~nm}$. Au vapors were deposited on the liquid surface, and adatoms could float around on the surface as Brownian movement. In addition, it could be considered that an adatom collides with another one at the surface, which results in the nucleation. The nucleation proceeds to form the critical nucleus, and the density of adatoms is proportional to the deposition rate. For the high deposition rate, the number of $\mathrm{Au}$ nuclei with a critical size increased as the deposition rate continued to increase. As a result, smaller clusters were formed at higher deposition rates because nucleation growth occurred in the ionic liquid.

ILs exhibit many features that are advantageous for a number of applications such as synthetic chemistry, catalysis, photochemistry, and electrochemistry [43-45]. For example, ILs are electrically conductive and can be applied to fuel cells as an electrolytic liquid. In order to improve the conductivity, Au clusters were formed in the ILs. Figure 7 shows the alternating current impedance of EMIM-DCA measured at different frequencies. When the IL includes Au clusters (nanoparticles), the real part of the impedance is smaller than that of the IL without $\mathrm{Au}$ clusters. This indicates that a more conductive IL is obtained when it contains Au clusters.

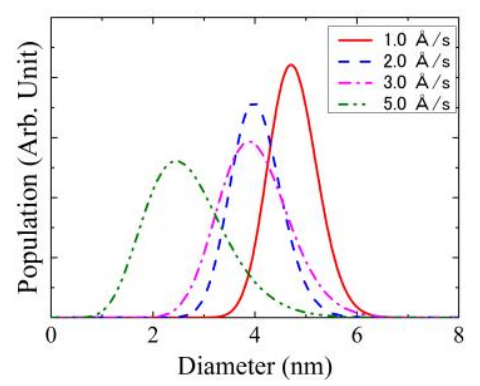

Fig. 6: Size distribution of the Au clusters formed in EMIM-DCA at different deposition rates.
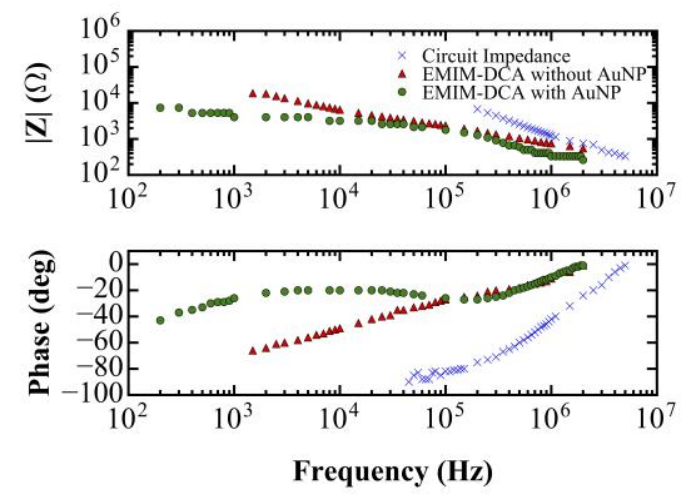

Fig. 7: Alternating current impedance $Z$ measured at different frequencies for EMIM-DCA with and without $\mathrm{Au}$ clusters.

\section{IRRADIATION EFFECTS OF CLUSTER IONS \\ 3.1 High-density irradiation effect}

Figure 8 shows a schematic of the impact of cluster ions on a solid surface $[27,46]$. The diameter of a cluster comprising several thousand molecules is a few nanometers, and these clusters penetrate the solid surface to a depth of only a few nanometers. As a result, multiple collisions occur between cluster molecules and surface atoms. Furthermore, the incident energy of a colliding cluster ion is transferred to the surface of the material. This dense energy deposition results in an increase in the cluster temperature, as well as increased impact area temperature. According to the molecular dynamics (MD) simulation for an Ar cluster ion impact on a $\mathrm{Si}(100)$ surface [20], the Ar cluster temperature reached approximately $40,000 \mathrm{~K}$ a few pico-seconds after impact, with an incident energy of $50 \mathrm{eV}$ per atom. In addition, the temperature of the impact surface area was approximately $2,000 \mathrm{~K}$

Light was emitted from substrates irradiated by water cluster ion beams. The luminescence was monitored in situ outside the vacuum chamber through an optical lens and light guide. The light intensity was measured using a photon counter (Hamamatsu H8259), which exhibits a 
sensitive response in the $185-850 \mathrm{~nm}$ wavelength range. The total optical efficiency was approximately $0.15 \%$ [47]. Figure 9 shows the relationship between the light intensity and acceleration voltage for the water cluster ions. The ion current density was $76 \mathrm{nA} / \mathrm{cm}^{2}$. As shown in the figure, luminescence was observed when the acceleration voltage was larger than $3 \mathrm{kV}$. The intensity increased with increasing acceleration voltage. The peak size of the water cluster ions was approximately 3,000 molecules and the average incident energy was a few electron volts per molecule. More detailed experiments on Ar cluster ion irradiation of $\mathrm{SiO}_{2}$ targets indicated that the cluster temperature was approximately $13,000 \mathrm{~K}$ at an acceleration voltage of $9 \mathrm{kV}$. This was estimated from the intensity ratio of the photoluminescence observed at different acceleration voltages [48].

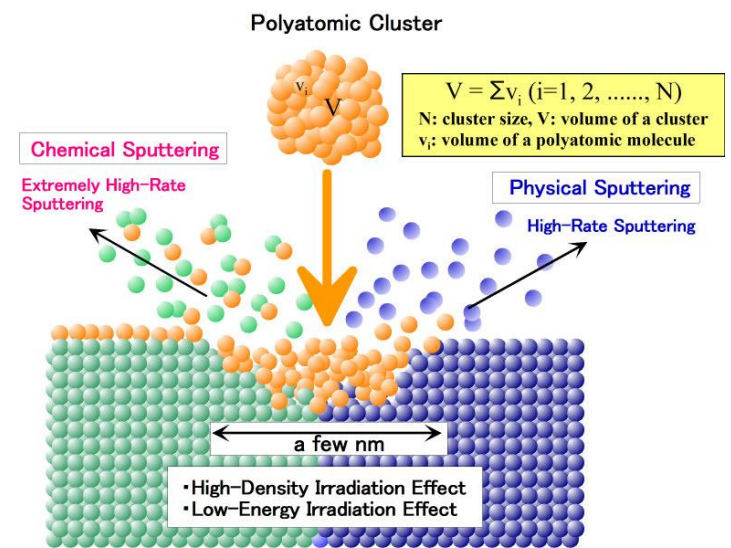

Fig. 8: Schematic illustration of the impact of cluster ions on a solid surface.

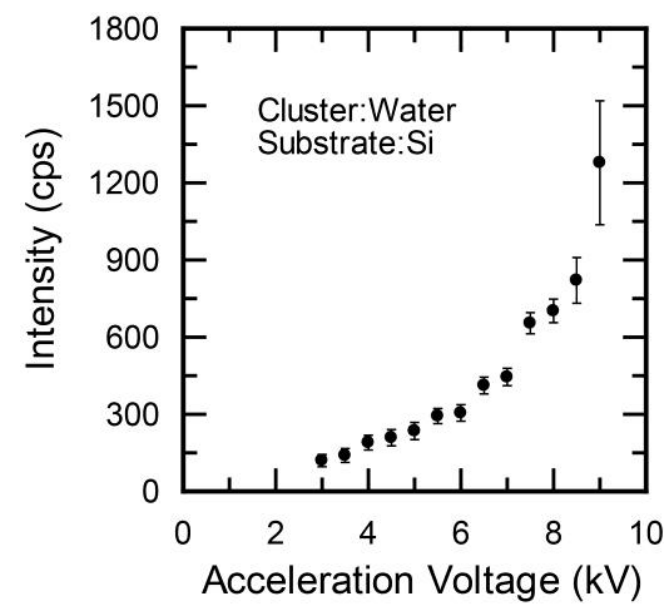

Fig. 9: Relationship between the light intensity and acceleration voltage for the water cluster ions.

\subsection{Low-energy irradiation effect}

The irradiation damage by the cluster ion beams was investigated by the Rutherford backscattering spectrometry (RBS) method. Figure 10 shows the number of displacement atoms for the $\mathrm{Si}(100)$ surfaces irradiated at different acceleration voltages by ethanol and water cluster ions and by Ar monomer ions. The ion dose was $1.0 \times 10^{16}$ ions $/ \mathrm{cm}^{2}$. The minimum size of the cluster ions was 100 molecules. As shown in the figure, the number of displacement atoms, that is the irradiation damage, increased with increasing acceleration voltage. For the Ar ion irradiation, the irradiation damage was much greater than that for the ethanol and water cluster ion irradiation. With regard to the cluster ion irradiation, the incident energy per molecule, which is the accelerating energy divided by the cluster size, was very low. Therefore, the irradiation damage induced by the cluster ion beams was less than that by the Ar monomer ion beams. Furthermore, at an acceleration voltage of $1 \mathrm{kV}$, the number of displacement atoms by the ethanol cluster ion irradiation was the same as that of the unirradiated Si surface. Because the incident energy of an ethanol molecule is less than $10 \mathrm{eV}$, a damage-free surface was obtained by extremely low-energy irradiation of the ethanol cluster ion beams. In addition, the incident energy could be changed to the thermal energy. For example, we measured the damage for Si surfaces irradiated at the incident energy of a few electron volts per atom. The damage decreased with increasing the fluence, and the thermal annealing effect was obtained [49].

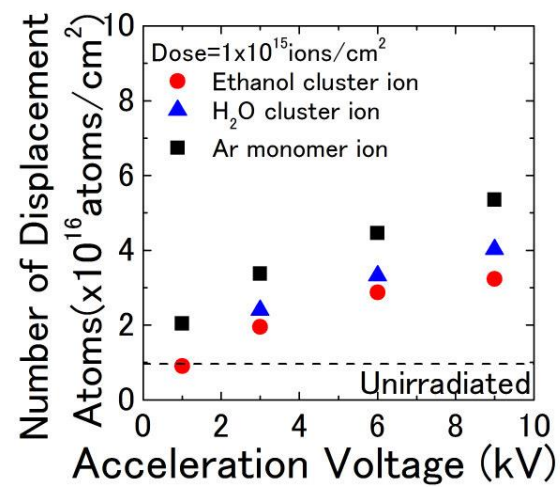

Fig. 10: Number of displacement atoms for the $\mathrm{Si}(100)$ surfaces irradiated at different acceleration voltages by ethanol and water cluster ions and by Ar monomer ions.

Furthermore, the irradiation damage by ethanol cluster ion irradiation at different retarding voltages was measured. The acceleration voltage was $6 \mathrm{kV}$, and the ion dose was $1.0 \times 10^{16}$ ions $/ \mathrm{cm}^{2}$. The number of displacement atoms decreased with increasing retarding voltage, and it decreased by one-third at a retarding voltage of $81 \mathrm{~V}$ as compared to $28 \mathrm{~V}$. This indicates that the irradiation damage decreases significantly when the retarding voltage increases, such as up to $150 \mathrm{~V}$.

\section{MATERIAL PROCESSING USING CLUSTER ION BEAMS}

4.1 Theoretical model for physical and chemical sputtering

The impact of cluster ions on solid surfaces occurs on nanoscale in a vacuum. As already shown in Fig. 8, the volume $V$ of a polyatomic cluster could be given by

$$
V=\sum v_{i}(i=1,2, \ldots \ldots, N)
$$

Here, $N$ is the cluster size (the number of polyatomic 
molecules) and $v_{i}$ is the volume of a polyatomic molecule contained in the cluster. The elements in volume $V$ are various particles such as atoms, molecules, and radicals contained in a cluster. The intersection of $V$ and $v_{i}$ denoted by $S$ plays a central role in the interaction of the cluster ion with solid surface atoms, and it is defined by [39]

$$
S=\sum V \cap \overline{v_{i}} \quad(i=1,2, \ldots \ldots, N)
$$

where $v_{i}$ is the complemental set of $v_{i}$. In addition, the momentum and total energy contained in the volume $V$ and $v_{i}$ are assumed to be constant prior to the impact of the cluster ion. After impact, the incident energy of a cluster ion is deposited at the solid surface, and this dense energy deposition results in the enhancement of cluster temperature, as well as the impact area temperature. Radicals, as well as excited atoms and molecules, are generated by dissociation of the polyatomic molecule. When the incident energy is higher than the threshold energy for sputtering, physical and chemical sputtering occur. In other words, physical sputtering occurs by the momentum transfer of cluster ions, and the sputtered atoms of the substrate surface are ejected into region $S$. In addition, for the chemical sputtering, chemical reactions such as oxidation and hydration occur on the substrate surface, and volatile products are evaporated into region $S$.

The rate of chemical reaction $(\eta)$ of multiple reaction paths is described as follows [50]:

$$
\eta \propto N_{d} \frac{k T}{h} \sum_{j=1}^{n} \exp \left(-\frac{Q_{j}}{k T}\right)
$$

where $N_{\mathrm{d}}$ is the number density of polyatomic molecules such as water molecules, $\mathrm{h}$ is the Planck constant, $\mathrm{k}$ is the Boltzmann constant, $T$ is the temperature of the polyatomic molecules after impact, $Q_{\mathrm{j}}(\mathrm{j}=1,2, \ldots \mathrm{n})$ is the activation energy for the $j$ th reaction path, and $n$ is the number of each reaction path. The equation indicates that the rate of chemical reaction increases with increasing temperature.

\subsection{Chemical modification and sputtering process}

Figure 11 shows the sputtering yields for $\mathrm{Al}, \mathrm{Si}, \mathrm{Ti}, \mathrm{Ni}$, $\mathrm{Cu}, \mathrm{Ag}$, and Au surfaces at an acceleration voltage of $9 \mathrm{kV}$ [27]. In the figure, the sputtering yields due to irradiation of Ar monomer ion beams at the same acceleration voltage are also shown, and these agree with the values estimated by computer simulations [51]. As shown in the figure, the sputtering yield due to water cluster ion irradiation was approximately 10 times larger than that caused by $\mathrm{Ar}$ monomer ion irradiation. In addition, selective sputtering on different metal surfaces takes place. When this occurs, the water cluster ion irradiation on metal surfaces causes physical sputtering. On the other hand, in the case of ethanol cluster ion irradiation, $\mathrm{Al}, \mathrm{Ti}$, and $\mathrm{Ni}$ surfaces, as well as Si surfaces, are sputtered more effectively, and the sputtering yield is approximately 100 times larger than that due to Ar monomer ion irradiation. This is thought to be due to the enhancement of chemical sputtering, in which alkyl compounds of the metal are formed as volatile products. Alkyl radicals such as $\mathrm{CH}_{2}, \mathrm{CH}_{3}, \mathrm{C}_{2} \mathrm{H}_{4}$, and $\mathrm{C}_{2} \mathrm{H}_{5}$ are included in an ethanol molecule, and are produced after the impact of ethanol cluster ions on the metal surface. These radicals play an important role in the chemical reactions of cluster ions with the atoms on the

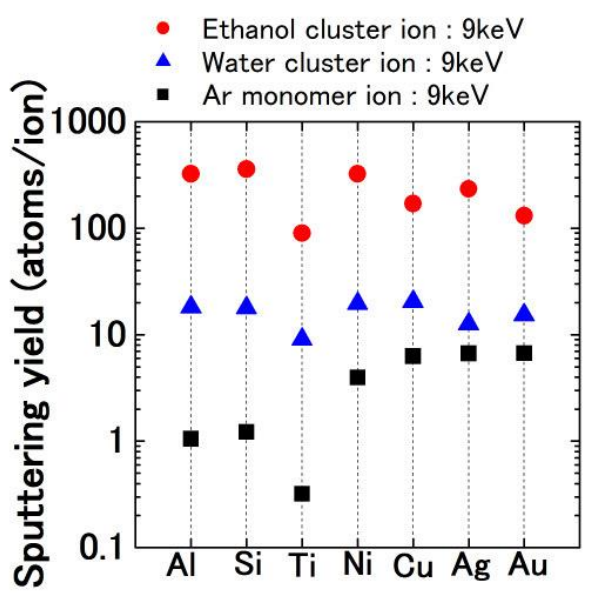

Fig. 11: Sputtering yields for various substrates irradiated at an acceleration voltage of $9 \mathrm{kV}$ by ethanol and water cluster ions and by Ar monomer ions.

metal surface. Furthermore, with regards to metals such as $\mathrm{Cu}, \mathrm{Ag}$, and $\mathrm{Au}$, the high rate sputtering occurred by the momentum transfer of incident atoms because the sputtering yields were similar to those for Ar cluster ion irradiation.

Figure 12 shows the sputtered depth of $\mathrm{Si}(100)$ and $\mathrm{SiO}_{2}$ surfaces irradiated by ethanol cluster ion beams at different retarding voltages. The acceleration voltage was adjusted to be at 3,6 , and $9 \mathrm{kV}$. As shown in the figure, the sputtered depth of the Si surface increases with increasing retarding voltage, and it has a peak at the acceleration voltage of $9 \mathrm{kV}$ and the retarding voltage of $112 \mathrm{~V}$, which corresponds to the minimum size of approximately 400 molecules. This is due to chemical sputtering, and the sputtered depth is significantly related to the implanted depth of the cluster ions. In other words, the implanted depth decreases with increasing minimum size of cluster ions, and the amount of volatile products increases with increasing retarding voltage. Furthermore, at higher retarding voltages, the incident energy per molecule decreases, and thus the chemical reaction decreases. On the other hand, for the $\mathrm{SiO}_{2}$ surface, the sputtered depth decreases with increasing retarding voltage. This is due to physical sputtering, and the sputtered depth decreases with the increasing minimum size of the cluster ions. However, for cluster beams with an incident energy less than a few electron volts per molecule, the accelerating energy changes to thermal energy after impact on the $\mathrm{SiO}_{2}$ surface. As a result, thermal annealing occurs on the surface.

Ion beams have several features, one of which is that the simultaneous use of several beams, e.g. electron and ion beams and monomer and cluster beams, are available. In addition, the simultaneous use of different energy beams has attracted much interest as an exotic ion beam, and it can be performed by adjusting the desired energy with cluster ion beams. For example, the simultaneous use of different processes, e.g. etching and annealing, is performed by adjusting the incident energy per molecule, which is the velocity. For physical sputtering, the energy range is larger than a few tens of electron volts per molecule. For chemical sputtering, it is a value between a 
few electron volts and a few tens of electron volts per molecule [27]. For energy less than a few electron volts per molecule, a damage-free region is achieved $[49,52]$. Therefore, no damage etching is expected by the cluster ion beam technique, which is not the case of conventional ion beam processes.
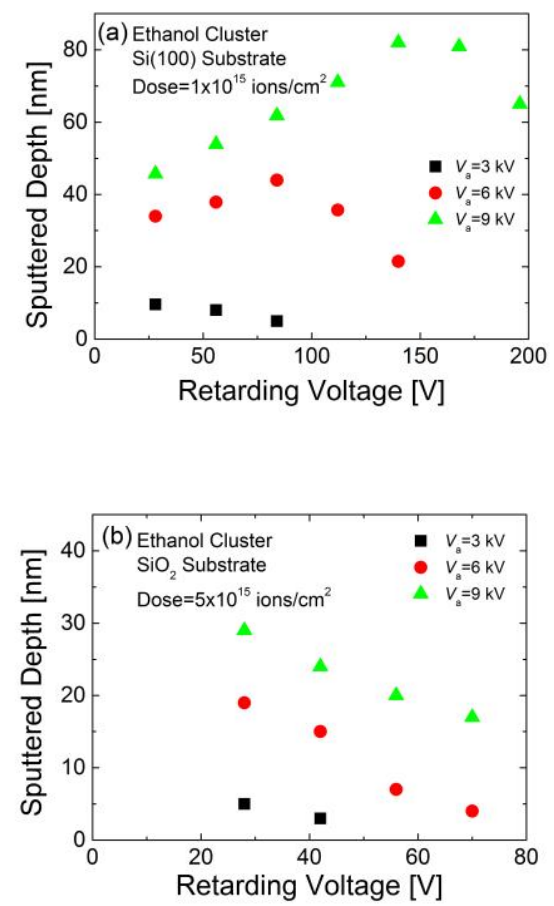

Fig 12: Sputtered depth of (a) $\mathrm{Si}(100)$ and (b) $\mathrm{SiO}_{2}$ surfaces irradiated at different retarding voltages by ethanol cluster ion beams.

\section{CONCLUSION}

Various kinds of clusters such as hydrogen, ionic, and metallic bonding clusters were generated using unique methods such as the nozzle beam, high-electric-field, and evaporation-on-liquid methods. The cluster size and structure of these clusters were analyzed by TOF, HEED, TEM, and SAXS. The size of water or ethanol clusters, which represented the hydrogen bond network structure, was in the range of approximately 100 to 10,000 molecules. The cluster size increased with increasing vapor pressure. With regards to the metallic bonding clusters, the average diameter of Au clusters was between approximately 1 and $6 \mathrm{~nm}$ depending on the evaporation conditions. Large clusters had an fcc crystal structure and small clusters had a decahedral structure, which was different from the fcc structure of bulk Au. For IL clusters, $(\mathrm{AB})_{n}$ consisting of cation $\mathrm{A}^{+}$and anion $\mathrm{B}^{-}$, the cluster size $n$ was between a few and a few tens of molecules. The positive and negative clusters were produced by applying a high voltage with a positive and negative polarity to the tip.

Additional functional materials were prepared based on liquids such as ILs, water, and ethanol. These ILs had unique properties, and the electric conductivity was enhanced by forming Au clusters in the IL (Emim-DCA). Gas hydrate clusters such as $\mathrm{CH}_{4}$ and $\mathrm{CO}_{2}$ were generated by introducing the gas into liquid water to induce cluster formation. In addition, liquid cluster ions had several beneficial features, one of which was that the impact on a solid surface represented unique irradiation effects such as low-energy and high-density irradiation effects. Furthermore, the kinetic energy of the cluster ion was converted to thermal energy, and extremely high temperatures were achieved. As a result, photo-luminescence was observed by water cluster ion irradiation.

The cluster ion beam technique is a material processing technique that uses exotic ion beams such as the simultaneous use of different processes, as well as different beams. The simultaneous use of chemical sputtering and thermal annealing processes was demonstrated with ethanol cluster ion beams. As a result, low irradiation damage and high-rate sputtering of $\mathrm{Si}$ surfaces was obtained by the retardation potential method.

\section{References}

[1] T. Takagi, Pure \& Appl. Chem., 60 (5) (1988) 781.

[2] E.Schumacher, CHIMIA, 42 (1988) 357.

[3] M.A.Duncan and D.H.Rouvray, Sci. Am., (1989) 110.

[4] M.L.Cohen and W.Knight, Phys. Today, (1990) 42.

[5] Ph. Buffat and J-P. Borel, Phys. Rev. A13 (1976) 2287.

[6] M.D.Morse, Chem. Rev., 86 (1986) 1049.

[7] W.D.Knight, W.A.de Heer and W.A.Saunders, Z. Phys., D3 (1986) 109.

[8] A.W. Castleman, Jr. and R.G. Keesee, Chem. Rev. 86 (1986) 589.

[9] I.Yamada and G.H.Takaoka, Jpn. J. Appl. Phys. 32 (1993) 2121.

[10] J. Ishikawa, Rev. Sci. Instrum. 79 (2008) 02 C506.

[11] J. Peters, Rev. Sci. Instrum. 79 (2008) 02A515.

[12] C.M. Lyneis, D. Leitner, D.S. Toda, G. Sabbi, S. Prestemon, S. Caspi and P. Ferracin, Rev. Sci. Instrum. 79 (2008) 02A321.

[13] D.J. Stokes, L. Roussel, O. Wilhelmi, L.A. Giannuzzi and D. HW Hubert, Mater. Res. Soc. Proc. 1020 (2007) 15.

[14] H. Yasumatsu and T. Kondow, Rep. Prog. Phys. 66 (2003) 1783.

[15] H. Haberland, "Clusters of Atoms and Molecules" (Springer-Verlag, Berlin, 1994)

[16] H. Hsieh and R.S. Averback, Phys. Rev. B42 (1990) 5365.

[17] R.S. Averback, M. Ghaly and H. Zhu, Radiat. Eff. Defects Solids 130-131 (1994) 211.

[18] Z. Insepov, I. Yamada and M. Sosnowski, Mater. Chem. Phys. 54 (1998) 234.

[19] M. Moseler, O. Rattunde, J. Nordiek and H. Haberland, Nucl. Instrum. Methods B164-165 (2000) 522.

[20] Z. Insepov and I. Yamada, Surf. Rev. Lett. 3 (1996) 1023.

[21] Z. Insepov and I. Yamada, Nucl. Instr. Methods, B121 (1997) 44.

[22] Y. Hatakeyama, M. Okamoto, T. Torimoto, S. Kuwabata and K. Nishikawa, J. Phys. Chem. C113 (2009) 3917.

[23] K. Richter, A. Birkner and A.-V. Mudring, Phys. Chem. Chem. Phys. 13 (2011) 7136.

[24] G.H.Takaoka, M. Kawashita and T. Okada, Mater. Res. Soc. Proc. 1020 (2007), p.159. 
[25] G.H. Takaoka, M. Kawashita and T. Okada, Rev. Sci. Instrum. 79, $02 \mathrm{C} 503$ (2008).

[26] H. Ryuto, K. Tada and G.H. Takaoka, Vacuum 84, 501 (2010) 501.

[27] G. H. Takaoka, H. Ryuto and M. Takeuchi, J. Mater. Res. 27 (2012) 806.

[28] G.H. Takaoka, H. Noguchi, K. Nakayama, Y. Hironaka, and M. Kawashita, Nucl. Instrum. Methods, B237 (2005) 402.

[29] G.H. Takaoka, K. Nakayama, T. Okada and M. Kawashita, Proc. 16th Int. Conf on Ion Implant. Technol., edited by K.J. Kirkby, R. Gwilliam, A. Smith and D. Chivers, (AIP Conf. Proc., 2006), p.321.

[30] G.H. Takaoka, M. Takeuchi, H. Ryuto and R. Ueda, Nucl. Instrum. Methods, B307 (2013) 257.

[31] M. Takeuchi, T. Hamaguchi, H. Ryuto and G.H. Takaoka,, Nucl. Instrum. Methods, B315 (2013) 345.

[32] G.H. Takaoka, Y. Matsumoto, M. Takeuchi and H. Ryuto, Proc. 10th Int. Symp. on Atomic Level Charact. (ALC'15), (2015) Art. No. 27p-P-46.

[33] O.F. Hagena, Phys. Fluids 17 (1974) 894.

[34] O.F. Hagena, Rev. Sci. Instrum. 63 (1992) 2374.

[35] E.D. Sloan, Nature, 426 (2003) 353.

[36] L.A. Stern, S.H. Kirby, W.B. Durham, Science, 273 (1996) 1843.

[37] A. Falenty, A.N. Salamatin, W.F. Kuhs, J. Phys. Chem. C117 (2013) 8443.

[38] G.H. Takaoka, H. Ryuto, M. Takeuchi and H. Kobayashi, Nucl. Instrum. Methods B326 (2014) 190.

[39] G.H. Takaoka, H. Ryuto, M. Takeuchi and F. Musumeci, Proc. 10th Int. Symp. on Atomic Level Charact. (ALC '15), (2015) Art. No. 26p-B-7.

[40] D.X. Zhang and R.G. Cooks, Int. J. Mass Spectrom. 196 (2000) 667.

[41] M. Gamero-Castano and J.F. de la Mora, Analy. Chim. Acta 406 (2000) 67.

[42] G.H. Takaoka, T. Hamaguchi, M. Takeuchi and H. Ryuto, Nucl. Instrum. Methods, B341 (2014) 32.

[43] K. Binnemans, Chem. Rev. 107 (2007) 2592.

[44] H. Zhao, J.E. Holladay, H. Brown and Z.C. Zhang, Science 316 (2007) 1597.

[45] N. V. Plechkova and K.R. Seddon, Chem. Soc. Rev. 37 (2008) 123.

[46] I. Yamada, Mat. Chem. Phys. 54 (1998) 5.

[47] H. Ryuto, F. Musumeci, A. Sakata, M. Takeuchi and G.H. Takaoka, Rev. Sci. Instrum. 85 (2014) 02 C 303.

[48] F. Musumeci, H. Ryuto, A. Sakata, M. Takeuchi and G.H. Takaoka, J. Lumines., 172 (2016) 224.

[49] G.H. Takaoka, H. Shimatani, H. Noguchi and M. Kawashita, Nucl. Instrum. Methods B232 (2005) 206.

[50] W.D. Kingery, H.K. Bowen and D.R. Uhlmann: "Introduction to Ceramics" (John Wiley \& Sons Inc., New York, 1976) Chap. 9.

[51] W. Eckstein: in R. Behrisch, W. Eckstein(Eds.) "Sputtering by Particle Bombardment, Top. Appl. Phys. 110 " (Springer, Berlin, Heiderberg, New York, 2007) p.33.

[52] I. Yamada, G.H. Takaoka, M. Akizuki, C.E. Ascheron and J. Matsuo, Proc. 10th Int. Conf. on Ion Implant. Technol., Catania, Italy (Elsevier Science B.V. 1995) p.1002.

(Received January 7, 2016; Accepted April 26, 2016;

Published Online September 1, 2016) 